\title{
An algorithm for braking curve calculations in ERTMS train protection systems
}

\author{
B. Friman \\ Friman Datakonsult AB and Uppsala University, \\ Human-Computer Interaction, Department of Information Technology, \\ Sweden
}

\begin{abstract}
The European Railroad Transportation Management Systems standard for train protection, ETCS, includes several advanced features for predicting the safe speed from a number of target locations ahead of the train. The braking system can have a different braking capability in different speed segments. The area in front of the train can contain a number of targets with different target and release speeds. The area in front of it is also segmented according to the gradients, in a way which is independent of the targets. The variables that shall be input to the braking curve algorithm therefore have three dimensions. Since permitted speed shall be calculated, rather than time to intervention, square roots are needed for the calculations, which require some computational power. The article suggests an algorithm where the gradients and the targets are combined in one table. This makes the input variable area two-dimensional instead of three-dimensional, which simplifies calculations and reduces the necessary number of square root calculations.
\end{abstract}

Keywords: ETCS, ERTMS, braking curve, ATP.

\section{Introduction}

The European Train Control System - the new European standard for automatic train protection, uses basically a three-dimensional set of data as input to the supervision algorithms. The three dimensions are: a table of speed restrictions, a table of gradient sections, and a table of deceleration abilities in relation to speed. The output from the algorithms is expressed in the form of speed values; e.g. which is the highest possible speed the train can run, in order to be able to 
obey all speed restrictions on the track ahead of the train? The three dimensions of data input may require a large number of calculations. The requirement for output to be expressed in the form of a speed, requires square roots to be used, which requires comparatively large computational power for each calculation, especially since real-time CPU'S are not normally equipped with dedicated floating point processors.

Enough computation power must be allocated to be able to supervise the maximum possible number of restrictions, gradients and deceleration segments, plus an ample safety margin, in order to satisfy the safety requirements of a train protection system. All calculations shall be repeated according to changes in input data, such as train position, train speed and when information about new speed restrictions becomes available.

This paper describes an algorithm using a two-dimensional approach for the calculation of the output speed values.

Section 2 will describe the basic requirements for train speed supervision in ETCS. In section 3, the two-dimensional approach and algorithm will be described. In section 4 , additions to this algorithm to be able to handle various special ETCS requirements will be described. Finally in section 5, the conclusions of this work will be summarized.

\section{Basic requirements for train speed supervision in ETCS}

The following is a simplified description of the requirements for train speed supervision in ETCS. Beside the following requirements, there are also other requirements such as separate service and emergency brake supervision, consideration to position measurement uncertainty, and numerous other requirements which are needed in ETCS, but which are not necessary for describing the basic algorithm for supervision of targets ahead of the train. Possible algorithmic solutions to some of the more detailed requirements are however discussed later in this article.

\subsection{Speed restriction table}

The speed restriction table can contain up to 31 restrictions ahead of the train (30 static speed restrictions and one distant signal speed restriction). (See $\mathrm{n}$ iter in packet 12 and packet 27 , in subset-026 chapter 7 and in subset-58.) The restriction speeds can vary between $0 \mathrm{~km} / \mathrm{h}$ and $600 \mathrm{~km} / \mathrm{h}$ in steps of $5 \mathrm{~km} / \mathrm{h}$. (See variable v_static and v_loa in packets 12 and 27 in subset 026 chapter 7 and in subset-058).

\subsection{Gradient section table}

The gradient section table can contain up to 31 sections defining the gradients on the track in front of the train. The gradient on one section can be between $-25,4$ and $+25.4 \%$ in steps of $0.1 \%$ (see variable $g_{-}$a om packet 21 in subset-026 chapter 7 and subset-058). 


\subsection{Deceleration ability table}

The deceleration table describes the trains' ability to brake, as a function of speed. The use of such a table makes it possible to brake later in certain situations, since the known differences in braking ability related to speed are taken advantage of. The table cannot be longer than 31 speed segments. Each speed segment defines the trains braking ability in a certain speed range. The braking ability can be between 0 and $2.55 \mathrm{~m} / \mathrm{s} / \mathrm{s}$ in steps of $0.01 \mathrm{~m} / \mathrm{s} / \mathrm{s}$.

\subsection{Brake delay time}

Two models for brake delay are allowed. One assumes that the braking ability is zero during the brake delay and $100 \%$ after the brake delay. The other model uses two delay intervals. The braking ability is assumed to be zero during the first interval. During the second interval, braking ability is assumed to be gradually increasing to $100 \%$. The algorithm described in this paper uses the first, simpler brake delay model.

\section{A two dimensional approach and supervision algorithm}

The supervision algorithm shall calculate a palette of speeds for various purposes, from the input data in form of speed restrictions, gradient sections, deceleration table and brake delay time. This palette includes:

- Service brake intervention speed SBI: Which is the highest speed that the train can run and still be able to obey all speed restrictions ahead of the train, using only the service brake?

- Emergency brake intervention speed EBI: Which is the highest speed that the train can run and still be able to obey all speed restrictions ahead of the train, using the emergency brake (which has significantly shorter brake delay time)?

- Warning speed $\mathrm{W}$ : Which is the highest speed that the train can run and the driver has still a few seconds margin before the SBI speed is exceeded (considering that the SBI speed becomes lower and lower when the train approaches a the start of a speed restriction)?

- Permitted speed P: Which is the highest speed that the train can run and the driver has still a few seconds margin before the $\mathrm{W}$ speed is exceeded (considering that the $\mathrm{W}$ speed becomes lower and lower when the train approaches a the start of a speed restriction)?

This article will describe an algorithm that can be used to calculate either of the above measures, depending on which brake delay time is used, which position uncertainty is added and which restriction margins are selected. A plausible strategy would be to first calculate the EBI and then use the same algorithm again to calculate SBI, $\mathrm{W}$ and $\mathrm{P}$, this time focusing on the restriction which showed to be most restrictive for EBI. The calculation of SBI, W and P can be done in one pass, so a total of two passed would then be necessary, where only on restriction would be considered in the second pass. 


\subsection{Basic steps in algorithm}

When calculating the trains braking ability towards a restriction, only the allowed speed and starting point of the restriction are of interest. These are in the following called targets. A target is thus a combination of a position and an allowed speed (beginning at that position). That implies that the restriction table is seen as a table of speed targets which the train must be able to brake to.

The first obvious step in the algorithm is to remove all targets which cannot possibly be the most restrictive target. That is the case when a more distant target allows the same or a higher speed than a closer target. After this first step, the target/restriction table will contain a down slope stair where each more distant target has a lower allowed speed than the previous. The first step is obvious and will not be further discussed in this article.

The second step in the algorithm is to merge the target/restriction table and the gradient table into one table which contains both target speeds and positions in the track where the gradient changes.

The third step in the algorithm is to calculate the brake delay distance, that is how long the train would run without any braking, given the train speed and the braking delay. Later in the article I will argue that why it is not the trains actual speed that shall be used to calculate the brake delay distance, but rather the previous result from using the algorithm (the highest possible speed which would make it possible to obey all targets in front of the train).

The fourth step in the algorithm is to calculate the allowed speed at all positions in the target/gradient table, starting with the last position and working stepwise backward towards the position of the train. At each step, a target speed to current allowed speed calculation is done. In this calculation, the gradient (which affects the trains braking ability) is fixed, since all gradient change positions are included in the table. The trains braking ability relative to speed may however be non-fixed. The trains' deceleration ability at the result speed may differ from the ability at the target speed. Therefore, this calculation is in itself divided into steps, one for each involved segment in the deceleration ability table. The resulting allowed speed shall be compare with the target speed at the new position if there is one and the lowest of both selected as allowed speed at that position. If there is not target speed at the new position (the new position represents a gradient change), then the result of the calculation shall be used as allowed speed at the new position.

The fifth step is to interrupt the calculations when the position becomes closer to the train than the brake delay distance, since the trains braking ability is assumed to be zero here. Targets on the distance were braking ability is zero, shall instead be directly compared with the calculated allowed speed, and the lowest value selected.

\subsection{Step 2: merging the targets/restriction and gradient tables}

The merging of the target/restriction and gradient tables can be illustrated by the following example: 
Table 1: $\quad$ Example of a target/restriction table.

\begin{tabular}{|l|l|}
\hline Position & Allowed speed \\
\hline $500 \mathrm{~m}$ & $100 \mathrm{~km} / \mathrm{h}$ \\
\hline $1000 \mathrm{~m}$ & $50 \mathrm{~km} / \mathrm{h}$ \\
\hline $1500 \mathrm{~m}$ & $0 \mathrm{~km} / \mathrm{h}$ \\
\hline
\end{tabular}

Table 2: $\quad$ Example of a gradient table.

\begin{tabular}{|l|l|}
\hline Position & Gradient up to this position \\
\hline $700 \mathrm{~m}$ & $0 \%$ \\
\hline $1200 \mathrm{~m}$ & $-1.0 \%$ \\
\hline $1700 \mathrm{~m}$ & $-2.0 \%$ \\
\hline
\end{tabular}

Table 3: $\quad$ Example of a merged target/restriction + gradient table.

\begin{tabular}{|l|l|l|l|}
\hline Position & Type & Allowed speed & Gradient up to this position \\
\hline $500 \mathrm{~m}$ & Restriction & $100 \mathrm{~km} / \mathrm{h}$ & - \\
\hline $700 \mathrm{~m}$ & Gradient change & - & $0 \%$ \\
\hline $1000 \mathrm{~m}$ & Restriction & $50 \mathrm{~km} / \mathrm{h}$ & - \\
\hline $1200 \mathrm{~m}$ & Gradient change & - & $-1.0 \%$ \\
\hline $1500 \mathrm{~m}$ & Restriction & $0 \mathrm{~km} / \mathrm{h}$ & - \\
\hline $1700 \mathrm{~m}$ & Gradient change & - & $-2.0 \%$ \\
\hline
\end{tabular}

\subsection{Step 3: calculating the brake delay distance}

The braking ability is assumed to be $100 \%$ closer the targets. But before a certain position, the braking ability is assumed to be zero. This position is calculated as the current position plus the brake delay multiplied by the train speed:

$$
\text { pos } s_{\text {delayend }}=\text { pos }_{\text {train }}+\text { brakedelay } \cdot v
$$

For the sake of knowing when to issue an automatic ATP brake, the actual train speed can be used in this algorithm. If however the speed result from the calculation shall also be used as information to the driver, then we must use the resulting speed from the calculation when calculating the brake delay distance. A larger train speed will result in a larger brake delay distance which in its turn will result in a lower allowed speed from the algorithm. This means that there is a circular dependence in the algorithm. Is this a problem? Assume that the allowed speed is calculated periodically as the train advances on the track. When the train approaches a target, the allowed speed will gradually decrease. If the algorithm used the result from the previous calculation when calculating the brake delay distance, then the dependence on previous cycles result may in this case cause a minor underestimation of the allowed speed which is showed to the driver. The error can be approximated as the trains braking ability during the time between two calculations. If, for example, the braking ability is $0.8 \mathrm{~m} / \mathrm{s} / \mathrm{s}$, and the calculations are done with $0.25 \mathrm{~s}$ intervals, then the error would be $0.7 \mathrm{~km} / \mathrm{h}$, and it would be in the safe direction. If for example a future restriction suddenly 
disappears, for example because a signal ahead of the train changes from stop to clear, then however the calculated allowed speed would be too high, because it would be based on a too short brake delay distance, and the error could be rather large. This problem can however be solved by limiting how much the allowed speed may increase from one calculation to the next, or by delaying the display of the new information until one the calculation has been performed two times (this could for example result in a delay of the increased speed display by $0.25 \mathrm{~s})$.

\subsection{Step 4: calculating the allowed speed at all positions in the table}

The basic formula for calculating the braking distance from one speed $\mathrm{v}_{1}$ to a lower speed $\mathrm{v}_{2}$ is:

$$
d b r=\frac{v_{1}^{2}}{2 \cdot d e c}-\frac{v_{2}^{2}}{2 \cdot d e c},
$$

where dec is the trains' deceleration ability, in $\mathrm{m} / \mathrm{s}^{2}$.

When dbr, dec and $\mathrm{v}_{2}$ are available, $\mathrm{v}_{1}$ can be calculated as:

$$
v_{1}=\sqrt{2 \cdot d b r \cdot d e c+v_{2}^{2}}
$$

So, if the allowed speed at position $\mathrm{p}$ is known, then the allowed speed at position $\mathrm{p}-1$ can be calculated as:

$$
v_{p-1}=\sqrt{2 \cdot\left(\text { pos }_{p}-p o s_{p-1}\right) \cdot d e c+v_{p}^{2}}
$$

The trains' deceleration is dependent of the gradient and of the train speed. However, we know that the gradient over the distance is fixed and available as the next gradient change in the table (in tablerow (p) or later).

The relation between gradient and deceleration is defined by the following formula:

$$
d e c=d e c_{0}+g \cdot \frac{g r a d}{100}
$$

where $\operatorname{dec}_{0}$ is the zero gradient deceleration, $g \approx 9.8186 \mathrm{~m} / \mathrm{s}^{2}$ (free fall acceleration) and gradient grad is expressed in \%. The formula can be easily understood if we consider the case when the $\operatorname{dec}_{0}$ is zero and the slope is $100 \%$. The deceleration would then become $-9.8186 \mathrm{~m} / \mathrm{s}^{2}$, which represents free fall acceleration.

As mentioned earlier, dec $_{0}$ is dependent on the trains' speed, in accordance with the deceleration ability table. If we assume that there is a function $f_{\text {vtodec }}$ available which looks up the table and returns the $\operatorname{dec}_{0}$ deceleration as function of train speed, then we know that the $\operatorname{dec}_{0}$ is equal to $f_{\text {vtodec }}(v p)$ at position $p \cdot \operatorname{dec}_{0}$ at position p-1 is however unknown at the moment, but we assume tentatively that it is the same as at position $\mathrm{p}$, and can then calculate dec as

$$
\text { dec }=f_{\text {vtodec }}\left(v_{2}\right)+g \cdot \frac{\operatorname{grad}_{p}}{100}
$$


Using dec above, $\mathrm{v}_{1}$ is now tentatively calculated. If $\mathrm{v}_{1}$ has the same deceleration $\operatorname{dec}_{0}$ as $\mathrm{v}_{2}$, then $\mathrm{v}_{1}$ is correct. To check this, we assume there is a function $\mathrm{f}_{\text {rangehigh }}$ which again looks up the deceleration ability table and returns the highest speed which has the same deceleration as its argument. The condition for $\mathrm{v}_{1}$ to be valid is:

$$
f_{\text {rangehigh }}\left(v_{2}\right) \geq v_{1}
$$

If this is not the case, then a stepwise process is used to calculate the valid $\mathrm{v}_{1}$. First we calculate the position where the allowed speed $=\mathrm{v}_{2}{ }^{\prime}=f_{\text {rangehigh }}\left(\mathrm{v}_{2}\right)$ using the basic brake distance formula (2) above:

$$
\operatorname{pos}_{v 2^{\prime}}=\operatorname{pos}_{p}-\left(\frac{v_{2}^{\prime 2}}{2 \cdot d e c}-\frac{v_{2}^{2}}{2 \cdot d e c}\right)
$$

where dec is calculated according to formula (6) above. We now know the allowed speed at position $\operatorname{pos}_{\mathrm{v} 2}$. We can then do a new tentative calculation of $\mathrm{v}_{1}$, again using equation (4) but now substituting $\mathrm{v}_{2}$ by $\mathrm{v}_{2}$ ' and $\operatorname{pos}_{\mathrm{p}}$ by $\operatorname{pos}_{\mathrm{v} 2}$. The new tentative $v_{1}$ is then compared with the new (higher) $f_{\text {rangehigh }}\left(v_{2}{ }^{\prime}\right)$. The process is continued until we reach a value $v_{1}$ which is $<=f_{\text {rangehigh }}\left(v_{2}{ }^{\prime}\right)$. Once the valid $\mathrm{v}_{1}$ is calculated it shall be compared to the target speed at the new pos $\mathrm{p}_{\mathrm{p}-1}$, if there is one. The lowest value shall be regarded as the allowed speed at $\operatorname{pos}_{\mathrm{p}-1}$. The process continues towards the train, and is interrupted when the end of the brake delay distance is reached.

\subsection{Step 5: to interrupt the speed calculation when the end of the brake delay distance is reached}

Since the braking ability is zero over the brake delay distance, the calculation of allowed speed shall be interrupted when the end of the brake delay distance is

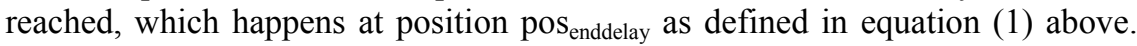
This means that if $\operatorname{pos}_{\mathrm{p}-1}<\operatorname{pos}_{\text {enddelay }}$, then pos enddelay shall be used instead of $\operatorname{pos}_{\mathrm{p}-1}$ in the last calculation according to step 4 . When the allowed speed at pos enddelay is calculated, this will not be updated anymore, except that it is replaced if there is a target speed which is lower (between the train and $\operatorname{pos}_{\text {enddelay }}$ ).

\section{Additions to the algorithm to be able to handle various special ETCS requirements}

In chapter 3, a basic algorithm to calculate the highest possible speed a train can run while still being able to obey the speed restrictions in the restriction table and considering the impact of gradients (the gradient table) and speed (the brake ability table) to the trains deceleration. In ETCS, and any other ATP system, there are many other requirements which need to be satisfied, e.g.

- The system shall be able to calculate allowed speed both for service brake and for emergency brake, the emergency brake being a minimum delay backup, should the service brake fail 
- Various speeds for informational purposes, such as permitted speed $\mathrm{P}$ and warning speed $\mathrm{W}$ shall also be possible to calculate

- Targets may have speed margins for different purposes (e.g. for service brake intervention and emergency brake intervention), and the supervision shall be interrupted when the target speed + speed margin is reached.

- A stop signal position ahead of the train may have a release speed associated with it, in order to make it possible for the train to reach the position where new signal information (possibly clear) becomes available. This is the case when transponders are used to convey the signalling information from the track to the train.

\subsection{Allowed speed for service brake and for emergency brake}

Service brake and emergency brake have different brake delays and their own deceleration ability tables. The allowed speed to avoid service brake and emergency brake can be calculated by running the above algorithm twice, once with the service brake delay and deceleration table and once with the emergency brake deceleration table. Another method which requires fewer calculations would be to first calculate the allowed speed to avoid emergency brake and then disable all restrictions except the one which was found to be most restrictive for the emergency brake before the algorithm is reused again for the service brake. If however, there is a stop signal among the targets, then this should always be included in the service brake calculations even if it is not the most restrictive emergency brake target, since such a target may be positioned significantly closer for service brake calculations than for emergency brake calculations (at least in ETCS). This is necessary in order to make sure that the train stops before the stop signal even when there is a large safety distance behind the signal.

\subsection{Various speeds for informational purposes}

In ETCS, the driver has to breach two speed limits before an automatic service brake is issued. The first limit is the permitted speed $\mathrm{P}$, which is showed to the driver during normal operation. As long as the driver runs the train below the $\mathrm{P}$ speed, he or she will have at least 5 second margin before ETCS would issue an automatic brake. If the driver exceeds the $\mathrm{P}$ speed with a certain margin, the ETCS system will issue a visible and audible warning to the driver. When ETCS issues the warning, the driver has still 3 seconds to react and start braking, before ETCS issues an automatic brake. Since $\mathrm{W}$ and $\mathrm{P}$ are defined in delay time units, they can be calculated by adding the $\mathrm{W}$ and $\mathrm{P}$ margins to the brake delay time before running the algorithm. To do this, two extra fictive delay end positions are calculated, one for $\mathrm{W}$ and one for $\mathrm{P}$ :

$$
\begin{aligned}
& p o s_{\text {enddelayw }}=p o s_{\text {enddelay }}+3 s . \cdot v \\
& p o s_{\text {enddelayp }}=p o s_{\text {enddelayw }}+2 s . \cdot v
\end{aligned}
$$

where $\mathrm{v}$ is the same speed as discussed in chapter 3.3. The calculations are carried out in one pass, but they are interrupted at different positions for the 
calculation of $\mathrm{W}$ and $\mathrm{P}$ than for the allowed speed to avoid service brake. This will cause $\mathrm{W}$ to be lower than the "allowed speed" and $\mathrm{P}$ to be lower than W, and it results in the desired time margins for the driver to react.

\subsection{Speed margins and release speeds}

For a signal speed or static speed restriction, there is a margin from the nominal speed to the speed when automatic service brake intervention is issued. There is also a margin between service brake intervention and emergency brake intervention. Unnecessary service brake intervention is thereby avoided as long as the driver drives close to the nominal speed, and unnecessary emergency brake is avoided when service brake is sufficient to do the job.

When the train approaches a restriction which has a margin, it is not necessary to brake the train down to the nominal speed - it is enough to brake it down to the nominal speed plus margin. Release speeds are similar to speed margins - the release speed is a speed margin above zero which enabled the train to approach the transponder close to the main signal.

Speed margins and release speeds are handled by calculating a position before the actual target where the allowed speed is equal to the target speed plus margin (or equal to release speed). The margin targets are used instead of the real targets in the calculations.

\section{Conclusion}

The ETCS requirements that gradients and targets shall be independently separated, and that the trains deceleration shall be defined in tables related to speed introduces considerable complexity in the supervision algorithms needed. This article suggests a method of combining the gradient and target tables, in order to master this complexity and to limit the necessary number of square root calculations. The allowed speed is calculated from the most distant target and backwards towards the position of the train. Fictive train delay times are used to produce the different speeds that are required for informational purposes.

\section{References}

[1] Alcatel, Alstom, ..., ERTMS/ETCS Class 1 System Requirements Specification, Chapter 7 ERTMS/ETCS language. SUBSET-026-7, Issue 2.2.2, Date 020201 .

[2] Alcatel, Alstom, ..., ERTMS/ETCS Class 1 System Requirements Specification, FFFIS STM Application Layer. SUBSET-058, Issue 2.1.1, Date 19-11-03. 